\title{
Radiology smartphone applications; current provision and cautions
}

\author{
M. A. Rodrigues • A. Visvanathan • J. T. Murchison • \\ R. R. Brady
}

Received: 2 April 2013 /Revised: 1 July 2013 / Accepted: 10 July 2013 / Published online: 4 August 2013

(C) The Author(s) 2013. This article is published with open access at Springerlink.com

\begin{abstract}
Objectives Medical smartphone applications are increasingly popular amongst doctors. However, the quality of their content is variable. We assessed contemporary radiologyrelated smartphone applications, focussing on the level of advertised medical involvement in application development. Methods Six major application stores were searched between 18-30 June 2012 using the terms radiology, radiation, $\mathrm{x}$-ray(s), computed tomography/CT, magnetic resonance imaging/MRI, ultrasound, nuclear medicine, fluoroscopy and mammography/mammogram. Application ratings, cost and medical input in development were recorded.

Results 321 applications were identified. One hundred fiftyeight were "teaching" and 96 "reference". Three of the 29 DICOM viewing applications had FDA approval for primary diagnosis, while $62 \%$ stated they should not be used for primary diagnosis; $24 \%$ of applications stated named medical professional involvement, $12 \%$ had unnamed medical involvement and $4 \%$ acknowledged guidelines or papers; $42 \%$ did not disclose authorship.

Conclusions A large variety of radiology-related smartphone applications are available with many potential benefits. Advertised medical involvement in application design is variable, making assessment of their accuracy difficult prior to purchase. Additional measures are required to ensure the accuracy of such applications. The limitations of image interpretation using
\end{abstract}

M. A. Rodrigues $(\triangle) \cdot J$. T. Murchison

Department of Radiology, Royal Infirmary of

Edinburgh, 51 Little France Crescent, Edinburgh, UK EH16 4SA

e-mail: mark.a.rodrigues@gmail.com

\section{A. Visvanathan}

Department of Infectious Disease, Western General Hospital,

Edinburgh, UK

R. R. Brady

Department of Surgery, Royal Infirmary of Edinburgh,

Edinburgh, UK smartphones are a major drawback of DICOM viewing applications. Further research into the accuracy of primary diagnosis using such applications is needed.

Main Messages

- A large variety of radiology smartphone applications are available with many potential benefits

- Variable medical involvement in application design limits assessment of accuracy before purchase

- Limitations of image interpretation using smartphones are a drawback of DICOM viewing applications

- Further work on the accuracy of primary diagnosis using these DICOM viewing applications is needed

Keywords Smartphone - Applications · Safety · Technology $\cdot$ Teleradiology

\section{Introduction}

Smartphone applications are software applications that can be downloaded onto smartphones. Smartphone health-related applications are rapidly increasingly in number, with approximately 1,000 new applications released each month [1] and 142 million annual downloads predicted by 2016 [2]. Their use is popular, with $85 \%$ of medical professionals using smartphones and 30-50\% reporting use of applications within their clinical practice $[2,3]$. The range of smartphone applications available has been reported in various specialties, including orthopaedics [4], colorectal surgery [5], anaesthesia/pain medicine [6, 7], and microbiology [8]. However concerns regarding the lack of medical involvement in application design $[5,7,8]$ and the reliability of application content $[9,10]$ have been raised.

The digital nature of modern radiology makes it ideally suited to smartphone applications. The potential utility of smartphone applications in radiology, such as portable Doppler ultrasound and mobile reporting of coronary computed 
tomography angiography $[11,12]$, have recently been reported; however, there is a general paucity of information within the literature regarding radiology-related applications. The Royal College of Radiologists of the UK (RCR) has recently launched "iRefer", an evidence-based and peer-reviewed radiological investigation guideline tool [13]. However the degree of medical involvement in the development of other radiology-related applications is currently unclear.

One of the most anticipated prospects in radiology is the development of smartphone applications that permit the mobile viewing of Digital Imaging and Communications in Medicine (DICOM) format images. This has the potential to allow remote reviewing of images, leading to potentially more timely diagnosis. However, the limitations of smartphone displays on primary diagnosis and the deleterious effects of other factors such as ambient light and network properties must be considered.

In view of this and given the popularity of smartphone applications in healthcare, we aimed to identify contemporary radiology-related smartphone applications, including teaching, reference and DICOM viewing applications, and assess the level of advertised medical involvement in application development.

\section{Materials and methods}

Six major application stores (Apple, Google Play, Blackberry Mobile Market, Nokia Ovi, Samsung and Microsoft Windows Marketplace) were searched for smartphone applications specifically targeted to radiology using the terms radiology, radiation, x-ray(s), computed tomography/CT, magnetic resonance imaging/MRI, ultrasound, nuclear medicine, fluoroscopy, mammography and mammogram. The searches were conducted by a single author between 18-30 June 2012 . Radiotherapy, veterinary and dental radiology applications as well as non-English language applications, games, wallpaper and applications under construction were excluded.

Data was recorded on the source of the application, application name, ratings, number of ratings and cost [prices in US dollars and British pounds were converted to euros using the exchange rate on 31 April $2013(\$ 1=€ 0.7779, £ 1=€ 1.1853)]$. Details regarding application content and medical input were obtained from the associated application description and any relevant websites.

Applications were categorised as "teaching" (flashcards, questions, revision guides/notes, textbooks, cases, tutorials/ talks, etc.), "reference" (guidelines, cancer staging, radiology positioning guides, scanning protocols, glossaries, calculators including radiation dose calculators, convertors, cost comparison, etc.), "viewing software", "Hospital Information System" (HIS) (patient data including imaging reports), "patient education" and "others" (marketing, magazines, journal access, etc.).
Food and Drug Administration (FDA) approval for viewing software applications was ascertained from applicationrelated publicity material and the FDA $510(\mathrm{k})$ premarket notification database [14].

Results were tabulated using Excel 2007 (Microsoft, USA). Where appropriate, two-tailed unpaired t tests were used to compare means.

\section{Results}

Three hundred twenty-one applications were identified (see Table 1 for a selection of example applications). The majority of these were available from either the Apple and Google Play application stores [172 (54\%) and $108(34 \%)$ respectively]. Six $(2 \%)$ were from the Blackberry Mobile Market and only 1 application was identified in each of the Nokia and Windows stores, while no relevant applications were found in the Samsung store. Twenty-one applications (7 \%) were available from both Apple and Google Play, ten (3\%) from Apple, Google Play and Blackberry, and two from Google Play and Blackberry stores.

\section{Categories of applications}

Table 2 displays a breakdown of application categories found on each source platform. "Teaching" was the most common category [158 (49\%)]. Teaching topics included patient cases (54), textbooks or encyclopaedias (40), exam revision aids (36) and radiological anatomy (30). "Reference" was the second most popular type (96 [30\%]), followed by "viewing software" (29 [9 \%]). Table 3 summarises the "viewing software" applications.

Medical involvement in application design

One hundred sixteen applications (36\%) had advertised medical professional involvement in application development. Seventy-six (24\%) of these stated specific named medical professional involvement (named doctor, radiographer, medical physicist or hospital) in the publicity information whilst a further $40(12 \%)$ mentioned unnamed medical professional involvement (such as "radiologist" or "emergency physician"). Twelve (4\%) acknowledged guidelines or papers used in developing the application, 12 (4\%) were designed by radiology or medical societies, such as the RCR or the American College of Radiology (ACR), and $37(12 \%)$ were developed by imaging companies (such as Siemens or General Electric). Two reported involvement of a named person but did not disclose their professional background, while six $(2 \%)$ stated general terms such as "exam experts"; 136 (42 \%) applications did not disclose authorship. 
Table 1 Examples of radiology-related smartphone applications. PACS=Picture Archiving and Communication System

\begin{tabular}{|c|c|c|}
\hline Application category & Example & Brief description \\
\hline \multirow[t]{2}{*}{ Teaching } & IMAIOS e-Anatomy & $\begin{array}{l}\text { Atlas of anatomy based on computed tomography and magnetic resonance images. } \\
\text { Users can scroll, zoom and rotate images, as well as toggle labels on and off }\end{array}$ \\
\hline & Radiopaedia & $\begin{array}{l}\text { Radiology cases for health professionals, with emphasis on radiology trainees. } \\
\text { Scrollable multi-image stacks, quiz questions and answers with links to related articles }\end{array}$ \\
\hline \multirow[t]{3}{*}{ Reference } & Virtual Radiographer & $\begin{array}{l}\text { Guide to radiographic positioning, with sample radiographs, radiographic calculator } \\
\text { and examples of common radiographic pathology }\end{array}$ \\
\hline & Lung TNM Calc & $\begin{array}{l}\text { Lung cancer staging calculator based on the } 7^{\text {th }} \text { Edition of the Lung Cancer } \\
\text { TNM Classification (2010) }\end{array}$ \\
\hline & iRefer & $\begin{array}{l}\text { Radiological investigation guidelines tool from the Royal College of Radiologists based } \\
\text { on the } 7^{\text {th }} \text { edition of "Making the best use of clinical radiology services". Helps referring } \\
\text { general practitioners, physicians and other health professionals determine the most } \\
\text { appropriate imaging investigation }\end{array}$ \\
\hline Viewer & eFilm Mobile & $\begin{array}{l}\text { Allows remote access to eFilm Workstations/PACS, permitting the user to view lists of studies, } \\
\text { forward studies to other eFilm devices, and view images on and download studies to a } \\
\text { smartphone. Not for diagnostic use }\end{array}$ \\
\hline Patient Education & Child MRI Prep & $\begin{array}{l}\text { Helps parents and medical personnel prepare children for MRI using pictures, sounds } \\
\text { and child-friendly language }\end{array}$ \\
\hline HIS & Ubikare & $\begin{array}{l}\text { Allows physicians to remotely access patient health records and test results, } \\
\text { including radiology, as well as view patient appointments }\end{array}$ \\
\hline Other & RSNA & $\begin{array}{l}\text { Official app of the Radiological Society of North America allowing access to mobile versions } \\
\text { of Radiographics, Radiology and RadiologyInfo. }\end{array}$ \\
\hline
\end{tabular}

\section{Cost of applications}

One hundred thrity-five applications were free to download, 4 provided free limited access to a paid application and 182 charged for access. The mean price of the paid applications was $€ 11.65$, ranging from $€ 0.82$ to $€ 82.97$. Of the paid applications, "viewing software" applications were the most expensive, costing on average $€ 27.11$ to download, followed by "teaching" (€13.08) and "reference" (€7.21).

Customer ratings

One hundred ninety-seven (61\%) applications were unrated. The mean rating of the 124 rated applications was 3.18 out of
5. Only $38(12 \%)$ applications had 10 or more ratings, and $10(3 \%)$ had more than 50 ratings. Paid applications rated significantly higher than free applications (3.65 versus 3.11 , $\mathrm{p}<0.0001$ respectively), as did those with medical input in design compared with no stated medical input (3.26 versus $3.07, \mathrm{p}<0.05$ respectively).

\section{Discussion}

Smartphones and their applications are increasingly used by medical practitioners and have potentially significant roles in the healthcare and clinical setting [15]. Health-related applications in various medical fields have been studied; however

Table 2 Number of radiology-related smartphone applications by category available from the different application stores. HIS=Hospital Information System

\begin{tabular}{|c|c|c|c|c|c|c|c|}
\hline Application store & Teaching & Reference & Viewing software & Patient education & HIS & Other & Total \\
\hline Apple & 91 & 47 & 18 & 1 & 2 & 13 & 172 \\
\hline Google Play & 52 & 35 & 5 & 1 & 3 & 12 & 108 \\
\hline Blackberry & 3 & 1 & 0 & 0 & 0 & 2 & 6 \\
\hline Nokia & 0 & 0 & 0 & 0 & 0 & 1 & 1 \\
\hline Windows & 0 & 1 & 0 & 0 & 0 & 0 & 1 \\
\hline Samsung & 0 & 0 & 0 & 0 & 0 & 0 & 0 \\
\hline Apple/Google Play & 6 & 7 & 6 & 0 & 0 & 2 & 21 \\
\hline Apple/Blackberry/Google Play & 6 & 3 & 0 & 0 & 0 & 1 & 10 \\
\hline Blackberry/Google Play & 0 & 2 & 0 & 0 & 0 & 0 & 2 \\
\hline Total & 158 & 96 & 29 & 2 & 5 & 31 & 321 \\
\hline
\end{tabular}




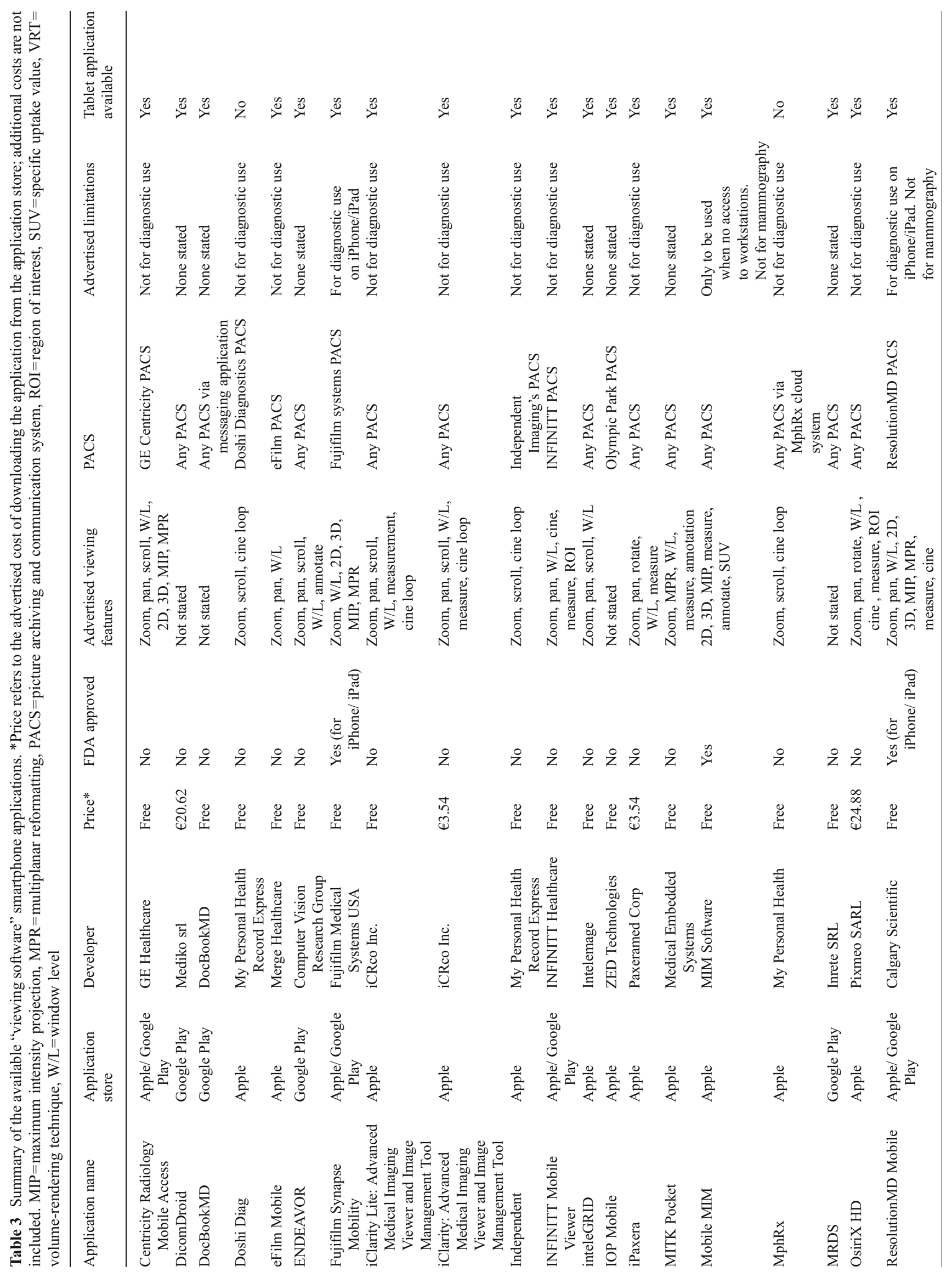




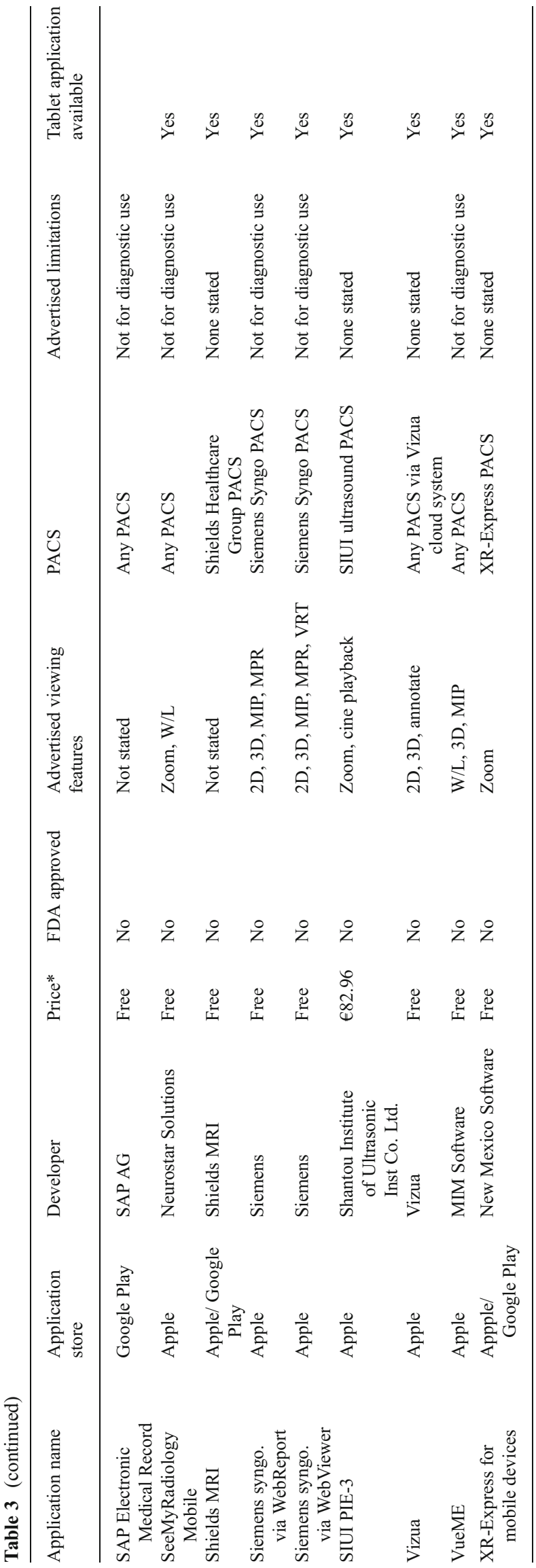

this is one of the first papers to review smartphone applications relevant to radiology.

Szekely et al. [16] recently published a similar study assessing the range and benefits of radiology-related smartphone and tablet applications. However their search only revealed 81 applications compared with the 321 identified in this study. Whilst the dates of the search and the exclusion criteria used differed between the studies, the search performed by Szekely et al. may have been incomplete.

Compared with the previous reports in other specialties, a relatively large number of radiology-related applications were identified. This probably reflects the general relevance of radiology compared with the previously studied medical fields, as well the rapid growth in medical applications [2].

The advent of smartphones and applications has made it easier for programmers, medically trained or not, to develop and publish medical-related literature. This increased accessibility has obvious benefits; however there are concerns regarding the accuracy of medical application content [5, 7-10]. In keeping with this, only $44 \%$ of applications in the study acknowledged medical professional/society involvement or the use of guidelines or papers in application development. A further $12 \%$ were developed by imaging companies. This low level of medical professional involvement is concerning.

It is currently difficult for a potential user to determine the accuracy of application content prior to purchase. The application stores provide a short description written by the application developer, customer ratings and comments, and a selection of screen shots. Whilst the customer ratings provide a source of feedback, $61 \%$ of applications in this study had no rating. Furthermore $69 \%$ of those rated were based on less than ten ratings, reducing the reliability of the customer feedback.

Expert medial input in the development of medical applications may improve reliability. This input should be clearly stated in the application advertising material to allow potential users to make an informed assessment of an application's reliability prior to purchase. Regulations may also be required for some medical applications. The FDA plans to regulate a subset of smartphone medical applications that might present a potential risk to patients, such as those allowing display of medical images directly from a picture archiving and communication system (PACS) server [17]; however most of the applications identified in this study are not part of this subset.

Another way of helping users gauge an application's reliability would be to develop a quality 'kite mark' for the field. This might be a role for the RCR or other radiology societies who could give their stamp of approval for products judged fit for the purpose.

With an appropriate application it is possible to access DICOM format images stored in PACS systems via a smartphone connected to the Internet. Fifteen out of the 29 viewing software applications have the facility to allow images from any PACS to be viewed remotely, and the 
remaining 14 permit access to a named PACS server. The viewing features available on these applications are becoming increasingly sophisticated, with many permitting multiplanar reformatting, two- or three-dimensional reconstructions, measuring as well as scrolling, panning, zooming and windowing.

The regulation of medical smartphone applications is a new and evolving field. The FDA has released guidance on its intention to regulate a subset of medical applications, including those allowing access to DICOM format images, however this guidance is currently only a recommendation as full regulatory oversight has not commenced [17]. Three of the viewing software applications in the study had FDA approval for primary diagnostic use (Table 3 ). The remaining 26 viewing software applications without FDA approval should not be used for primary diagnosis; however this was only stated in the publicity information of $62 \%$ of these.

To gain FDA approval the application must be shown to have substantial equivalence to another legally marketed device (i.e. any new technological changes are at least as safe and effective as the predicate). Whilst the software of the mobile PACS applications is very similar or identical to existing PACS systems, the relevant hardware is clearly different (smartphones versus reporting workstation), and this potentially affects the safety of the smartphone application. With these hardware limitations in mind both the Mobile MIM and ResolutionMD applications have clear disclaimers stating they should only be used when there is no access to a workstation, as they are not intended to replace full workstations.

The Medical Devices Directive (MDD) [18] ensures safety and performance of medical devices throughout the EU, with each member state having its own regulatory framework. However there is currently no consensus between member states over the definition of a medical device and which medical applications should be considered medical devices, leading to uncertainty among application developers and healthcare providers. In the UK, DICOM viewing applications would be considered a medical device by the Medicines and Healthcare Products Regulatory Agency (MHRA). Every registered medical device in the EU must carry the $\mathrm{CE}$ (Conformité Européene) mark, signalling that the manufacturer is satisfied the product conforms to the relevant requirements in the relevant legislation. The necessary requirements vary according to the MDD's classification of the medical device. Most applications, including DICOM viewing applications, are within the lowest risk category (class I), which means the developers can self-declare conformity to the essential requirements without the need for independent review. Unfortunately there is currently very limited use of the CE mark in the application stores [10].

The ability to view full-resolution images remotely has implications for timely diagnosis and treatment; however the limitations of using smartphones for image interpretation must be considered [19]. First, there are few studies investigating the diagnostic accuracy of image interpretation using smartphones [12, 20-23]. Second, smartphones cannot meet the minimum standards for primary diagnostic displays required in Europe. For example, the RCR requires a screen resolution of $\geq 1,280 \times 1,024$ pixels and screen size of $\geq 42$ $\mathrm{cm}$, with the possibility of comparing serial examinations side by side [24]; the iPhone 5's display for example has $1,136 \times 640$ pixels and a $10.2-\mathrm{cm}$ screen [25]. The deleterious effects of ambient light on mobile image interpretation also need to be taken into account. Finally, reporting workstation displays undergo rigorous regular calibration and quality assurance procedures to ensure they remain fit for the purpose. Smartphones being used for primary diagnosis would have to be part of similar quality assurance programmes.

Therefore, despite FDA approval, caution must be used when using DICOM viewing applications for primary diagnosis on smartphones and we feel the fundamental limitations of smartphone displays means smartphones are more suited for reviewing images. Whilst radiologists are likely to appreciate such restrictions, other medical practitioners using these applications may be less aware of their limitations.

In contrast to smartphones, tablet devices have larger displays with better resolution. They may therefore be better suited for mobile review of DICOM images. Studies assessing tablet devices in this regard are promising [26-28]; however further research is required to determine their diagnostic accuracy.

Data confidentiality is a major concern when using smartphones to review patient images, particularly if the phone is lost or stolen. One option used by several of the viewing applications is not storing any protected health information on the mobile device. Instead the user has to $\log$ in to a secure server and access the data via a secure, encrypted data transfer link. Further improvements in data security can be gained from applications utilising the HTLM5 markup language. In contrast to previous versions, HTLM5 has many commonly used applications integrated, such as video and audio elements. This makes it easier for users to access graphical and multimedia content directly via the web server without the need for additional plug-ins and subsequent data download onto a user's device. Whilst these options provide safe methods for managing confidential data, it obviously limits the use of the application to areas that have Internet access. Other applications permit the download of protected health information onto smartphones. Data stored in this way should be encrypted, and there should be the possibility to remotely disable lost or stolen devices.

There are other security concerns that should be mentioned. Employees, including healthcare workers, are increasingly using their own smartphones for work, resulting in a "bring your own device" trend. A recent survey showed that $41 \%$ of US healthcare workers do not have a password on their phone, over $50 \%$ access unsecure Wi-Fi networks and $48 \%$ have not disabled the Bluetooth discoverable mode [29]. Furthermore many healthcare institutions do not have a 
formal mobile strategy, with most unable to wipe the data from an employee's personal device should it be lost or stolen. Such practices raise serious security issues, which, in the setting of the healthcare system, could place patients' protected health information at risk.

The speed of data transfer and download is a further limitation of mobile image review on smartphones. A study evaluating the OsiriX smartphone application showed that the time taken to transfer $855-\mathrm{mm}$ axial slices of a CT of the abdomen and pelvis using an iPhone $3 \mathrm{~S}$ was up to $51 \mathrm{~s}$ with a WiFi connection, over 7 min using a $3 \mathrm{G}$ connection and over 35 min using an EDGE network connection [20]. The time taken for transferring thin (1-mm) slices to permit multiplanar formatting will be considerably longer. While smartphone and network technology continues to improve, with a corresponding increase in the rate of data transfer, the prolonged transfer times, particularly when no Wi-Fi connection is available, may currently limit the usefulness of smartphone viewing software applications.

Other concerns regarding noise, distraction, infection control, and health and safety cautions have also been reported $[30,31]$.

The advertised costs of some of the DICOM viewing applications are misleading. Some applications charge a one-off fee, which allows the user to download and use the application. In contrast, several applications are free to download but require access to a named PACS system or specific additional hardware or software to work. These "hidden fees" are often considerable, totalling hundreds or thousands of euros. The use of such applications is restricted to practitioners whose institutions have access to the required PACS system, negating some, if not all, of these "hidden fees", but restricting their wider usage.

One of the limitations of the study was the use of named medical involvement as a surrogate marker for reliability of application content. As the cost of downloading and reviewing all of the applications identified was prohibitively expensive, the validity and accuracy of the content within applications could not be directly verified. However, this recreates the situation potential application purchasers currently encounter prior to purchase.

\section{Conclusion}

Radiology-related smartphone applications are becoming increasing available and have many potential benefits. The most common applications are teaching and reference based, for which quality of information is crucial; however many of these do not appear to have medical input in their development. Additional measures, such as a quality 'kite mark', are advised to ensure accuracy of such application content and better inform users prior to purchase. Smartphone applications can also permit mobile interpretation of DICOM format images; however the limitations of smartphone displays and the need for optimal viewing conditions must be emphasised, especially if they are potentially used for primary interpretation.

Disclosures Richard Brady is owner of ResearchActive.com, a company that develops medical applications and provides mHealth solutions. No funding was received for this work.

Open Access This article is distributed under the terms of the Creative Commons Attribution License which permits any use, distribution, and reproduction in any medium, provided the original author(s) and the source are credited.

\section{References}

1. Sherwin-Smith J, Pritchard-Jones R (2012) Medical applications: the future of regulation. Ann R Coll Surg Engl 94:12-13

2. Cox A (2011) Mobile Healthcare Opportunities Interactive Forecast Suite. Smartphone Applications, Monitoring \& mHealth Strategies 2011-2016. Juniper Research. November 2011

3. Franko OI, Tirrell TF (2012) Smartphone app use among medical providers in ACGME training programs. J Med Syst 36:3135-9

4. Ol F (2011) Bhola S. iPad applications for orthopedic surgeons. Orthopedics 34:978-981

5. O'Neill, Brady RR (2012) Colorectal smartphone applications: opportunities and risks. Colorectal Dis 14:e530-4

6. Kraidin J, Ginsberg SH, Solina A (2012) Anesthesia applications: overview of current technology and intelligent search techniques. J Cardiothorac Vasc Anesth 26:322-326

7. Rosser BA, Eccleston C (2011) Smartphone applications for pain management. J Telemed Telecare 17:308-312

8. Visvanathan A, Hamilton A, Brady RR (2012) Smartphone applications in microbiology - is better regulation required? Clin Microbiol Infect 18:e218-20

9. Rodrigues MA, Brady RR (2011) Anaesthetists and applications: content and contamination concerns. Anaesthesia 66:1172-1185

10. Haffey F, Brady RRW, Maxwell S (2013) A comparison of the reliability of smartphone apps for opioid conversion. Drug Saf 36:111-117

11. Huang CC, Lee PY, Chen PY, Liu TY (2012) Design and implementation of a smartphone-based portable ultrasound pulse-wave Doppler device for blood flow measurement. IEEE Trans Ultrason Ferroelectr Frq Control 59:182-188

12. LaBounty TM, Kim RJ, Lin FY, Budoff MJ, Weinsaft JW, Min JK (2010) Diagnostic accuracy of coronary computed tomography angiography as interpreted on a mobile handheld phone device. JACC Cardiovasc Imaging 3:482-490

13. The Royal College of Radiologists (2012). RCR Referral Guidelines. United Kingdom. Available via: http://www.rcr.ac.uk/content.aspx? PageID $=995$. Accessed 8 July 2012

14. US Food and Drug Administration (2012) 510(k) Premarket Notification. US Food and Drug Administration. USA. Available via: http://www.accessdata.fda.gov/scripts/cdrh/cfdocs/cfpmn/pmn.cfm. Accessed 29 September 2012

15. Terry M (2010) Medical applications for smartphones. Telemed J E Health 16:17-22

16. Szekely A et al. (2013) Smartphones, tablets and mobile applications for radiology. Eur J Radiol. doi:10.1016/j.ejrad.2012.11.034

17. US Food and Drug Administration (2011) Draft guidance for industry and food and drug administration staff - mobile medical application. US Food and Drug Administration. USA. Available via: http:// 
www.fda.gov/MedicalDevices/DeviceRegulationandGuidance/ GuidanceDocuments/ucm263280.htm\#b. Accessed 8 July 2012

18. Medicines and Healthcare products Regulatory Agency (2013) Medical Device Directive 93/42/EEC of 14 June 1993. Available via: http://eur-lex.europa.eu/LexUriServ/LexUriServ.do?uri=CONSLEG: 1993L0042:20071011:en:PDF. Accessed 22 June 2013

19. Flanders AE, Wiggins RH 3rd, Gozum ME (2003) Handheld computers in radiology. Radiographics 23:1035-1047

20. Choudhri AF, Radvany MG (2011) Initial experience with a handheld device digital imaging and communications in medicine viewer: OsiriX mobile on the iPhone. J Digit Imaging 24:184-189

21. Yaghmai V, Kuppuswami S, Berlin JW, Salehi SA (2003) Evaluation of personal digital assistants as an interpretation medium for computed tomography of patients with intracranial injury. Emerg Radiol 10:87-89

22. Johnston WK 3rd, Patel BN, Low RK, Das S (2005) Wireless teleradiology for renal colic and renal trauma. J Endourol 19:3236

23. Toomey RJ, Ryan JT, McEntee MF, Evanoff MG, Chakraborty DP, McNulty JP et al (2010) Diagnostic efficacy of handheld devices for emergency radiologic consultation. Am J Roentgenol 194:469-474

24. The Royal College of Radiologists (2008) Picture archiving and communication systems (PACS) and guidelines on diagnostic display devices. The Royal College of Radiologists, London
25. Apple Inc (2012) iPhone. Apple Inc. USA. Available via: http:// www.apple.com/iphone/specs.html. Accessed 28 September 2012

26. John S, Poh AC, Lim TC, Chan EH, le Chong R (2012) The iPad tablet computer for mobile on-call radiology diagnosis? Auditing discrepancy in CT and MRI reporting. J Digit Imaging 25:628-34

27. Johnson PT, Zimmerman SL, Heath D, Eng J, Horton KM, Scott WW et al (2012) The iPad as a mobile device for CT display and interpretation: diagnostic accuracy for identification of pulmonary embolism. Emerg Radiol 19:323-7

28. McLaughlin P, Neill SO, Fanning N, McGarrigle AM, Connor OJ, Wyse G et al (2012) Emergency CT brain: preliminary interpretation with a tablet device: image quality and diagnostic performance of the Apple iPad. Emerg Radiol 19:127-33

29. CISCO mConcierge (2013) BYOD insights 2013: A CISCO partner network study. CISCO mConcierge. USA. Available via: http://www.ciscomcon.com/sw/swchannel/registration/internet/ registration.cfm?SWAPPID=91\&RegPageID=350200\&SWTHEMEID= 12949\&traffictype $=$ Direct. Accessed 8 June 2013

30. Brady RR, Verran J, Damani NN, Gibb AP (2009) Review of mobile communication devices as potential reservoirs of nosocomial pathogens. J Hosp Infect 71:295-300

31. Visvanathan A, Gibb AP, Brady RR (2011) Increasing presence of mobile communication technology: avoiding the pitfalls. Telemed $\mathrm{J}$ E Health 17:656-61 